\title{
Ferromagnetism driven by fluorine interstitials in MgO: A first-principles prediction
}

\author{
Hui Zhao ${ }^{a}, Y^{2}$ an Zhao ${ }^{b}$, \\ Department of Physics, Anshan Normal University, Anshan 114007, China \\ aemail: hzhao@aliyun.com,_bemail: 971967125@qq.com,
}

Keywords: first-principles; MgO; fluorine interstitial; ferromagnetism

\begin{abstract}
Our first-principles calculations based on density functional theory predicted a new path for the formation of sp-ferromagnetic states: anion interstitials. The fluorine interstitials in MgO were found to be spin polarized and are more stable in the ferromagnetic state. This observation is attributed to the strong p-p interaction between the interstitial $\mathrm{F}$ and the neighboring $\mathrm{O}$ atoms. We demonstrate that F-doped $\mathrm{MgO}$ is potential p-type half-metallic ferromagnets, and the source of magnetism is the spin triplet state splitting associated with p-p coupling, and ferromagnetism between interstitial atoms in these systems can be mediated by spin polarized holes.
\end{abstract}

\section{Introduction}

Ferromagnetic semiconductors or insulators have been attracting intense interest for their potential applications in the field of spintronic devices. ${ }^{1,2}$ Ideal ferromagnetic semiconductors or insulators should exhibit ferromagnetism at room temperature for practical applications and have a homogeneous distribution of the dopants. Over the past several years, there have been considerable interests in the possibility of creating ferromagnetism in thin films of doped oxide materials. Several systems have been identified to be ferromagnetic at room temperature but all of these systems contain either a transition metal (TM) element or a rare earth metal element as a dopant. ${ }^{3-7}$ Since in such systems the formation of nonrandom alloys, secondary phases or clusters of intrinsic magnetic ion precipitates cannot be rule out, the origin of room temperature ferromagnetism (RTF) is still controversial as well as the applications of these materials would be detrimental. ${ }^{8-10}$

In recent years, unexpected room temperature ferromagnetism was indeed observed in a series of materials which do not contain ions with partially filled $d$ or $f$ bands both experimentally and theoretically. ${ }^{11-14}$ This type of " $d^{0}$ ferromagnetism" provides a new opportunity for searching room temperature spintronic materials. As a common feature, defects with $s p$-type orbital character carry the magnetic moment in these systems. The increasing interest is not only due to the perhaps unexpected finding that $p$ bands can spontaneously polarize giving a ferromagnetic state but also due to high Curie temperature that are achieved even at low concentrations. Recently, Peng et al. pointed out that a threshold hole concentration is necessary to induce RTF. Therefore, to enhance RTF, one way is to introduce holes by stabilizing some native defects, impurities and complexes. Up to now, theoretical considerations have revealed mainly three routes for the formation of $s p$-ferromagnetic states by hole doping. In the first, $s p$-ferromagnetism can be achieved by cation vacancies. ${ }^{15-17}$ The main characteristic of the vacancies in all the systems is the high symmetry, either octahedral or tetrahedral, around the vacancy site. This invariably leads to a highly degenerate single particle spectrum, which may then present high-spin states. Secondly, the semiconductor or insulator cation is substituted by an atom of smaller valency, thus depriving the $p$-type valence band from electrons. The Fermi level $E_{\mathrm{F}}$ would be driven into the valence band deep energy level that the Stoner criterion is fulfilled and spontaneous spin polarization appears. The third scenario is that the anion is substituted by one of smaller valency, introducing shallow, spin-polarized gap states. As the impurity concentration increases, these states create impurity bands in the gap, which remain spin polarized if the Stoner criterion is still fulfilled. In the present letter, we predicted a new path for the formation of $s p$-ferromagnetic states: anion interstitials, which also could induce hole doping. 
The optimization of geometrical structures and the analysis of electronic structures and magnetic properties are performed using the calculation module CASTEP of Materials Studio 4.4 based on spin-polarized density functional theory (DFT). ${ }^{18}$ The generalized gradient approximation (GGA) in the scheme of Perdew, Burke and Ernzerhof (PEB), which is supposed to be "superior" for magnetic systems, is used for treatment of exchange-correlation interaction. ${ }^{19}$ The binary oxides of $\mathrm{Mg}$ adopts octahedral coordination in the cubic crystal system B1 rocksalt structure with space group FM-3M. In the letter, rocksalt $\mathrm{MgO}$ in the cubic crystal system are studied. Based on the experimental lattice constants, the systems were modeled with periodic supercells of $2 \times 2 \times 2$ with 64 atoms, which are sufficient to avoid the interaction of the $\mathrm{F}$ atoms with its images in neighboring supercells. Convergence with respect to the plane-wave cutoff energy and $k$-point sampling has been carefully checked. An energy cutoff of $380 \mathrm{eV}$ was used for the plane wave expansion of the electronic wave function. For the sampling of the Brillouin zone, special $k$ points were generated with a $3 \times 3 \times 3$ grid based on the Monkhorst-Pack scheme. ${ }^{20}$ Good convergence was obtained with these parameters, with the energy change to $5 \times 10^{-6} \mathrm{eV} /$ atom, maximum force to $0.01 \mathrm{eV} / \AA$, maximum stress to $0.02 \mathrm{GPa}$, and maximum displacement tolerances to $5 \times 10^{-4} \AA$, respectively. The atomic configurations used to generate the ultrasoft pseudopotentials are $2 p^{6} 3 s^{2}$ for $\mathrm{Mg}, 2 s^{2} 2 p^{4}$ for $\mathrm{O}$, and $2 s^{2} 2 p^{5}$ for $\mathrm{F}$, respectively.

The geometrical structures of conventional cell are optimized and compared with experimental values in order to confirm our calculations. The optimized lattice constants $\left(a_{0}=b_{0}=c_{0}\right)$ and the corresponding experimental values $\left(a_{e}=b_{e}=c_{e}\right)$ are $a_{0}=4.30 \AA$ and $a_{e}=4.22 \AA$ for MgO. ${ }^{21}$ Through analysis of these optimized and experimental lattice constants, the difference between the optimized lattice constants and the experimental values is $1.90 \%$ for $\mathrm{MgO}$. This means that our calculations are well in agreement with experimental values.

We consider the type of fluorine doping: fluorine interstitial (FI). For rocksalt MgO, there are two types of high-symmetry interstitial positions: tetrahedral $(T)$ and octahedral $(O)$ sites. ${ }^{22,23}$ The $T$ site corresponds to the center of the cube. It has eight nearest neighbors (four cations and four anions). The $O$ site is located at the center of the square. It has four nearest neighbors (two cations and two anions) and eight next-nearest neighbors (four cations and four anions). However, from our calculations, obtaining accurate results for the atomic configuration show that $O$ site interstitial is very difficult to be form due to the fairly large lattice relaxations induced by this defect.

Now we address the question of whether the presence of fluorine interstitial could possibly induce magnetism. The density of states (DOS) of the fluorine and its neighboring atoms for four kinds of interstitials are calculated and plotted in Fig. 1. These figures show the doped fluorine interstitials significantly change the DOS near the Fermi level and lead to the spin polarization of the valence band. Asymmetrical distributions of the DOS between the majority-spin and minority-spin channels mean the magnetism of such doped system. The strong coupling between $\mathrm{F} 2 p$ and $\mathrm{O} 2 p$ orbitals near the Fermi level can be seen clearly. The interaction causes the F $2 p$ state and the O $2 p$ state to split. The majority-spin bands are fully occupied while the minority-spin bands are partially filled, resulting in a magnetic moment of $1 \mu_{\mathrm{B}}$ per supercell. Based on spin population analysis, it is found that the magnetic moment is mainly contributed by the $2 p$ orbital of the $\mathrm{F}$ atom and the neighboring $\mathrm{O}$ atoms, while the second nearest neighboring $\mathrm{O}$ atoms also contribute a small part, suggesting the F-O system as the origin of magnetism in F-doped group-II oxides. The estimated formation energy of the $F_{I}$ defect is $1.7838 \mathrm{eV}$. The smaller the formation energy, the easier the dopants incorporation into the host crystal lattice; this indicates that the kind of interstitials may be realized easily experimentally. 


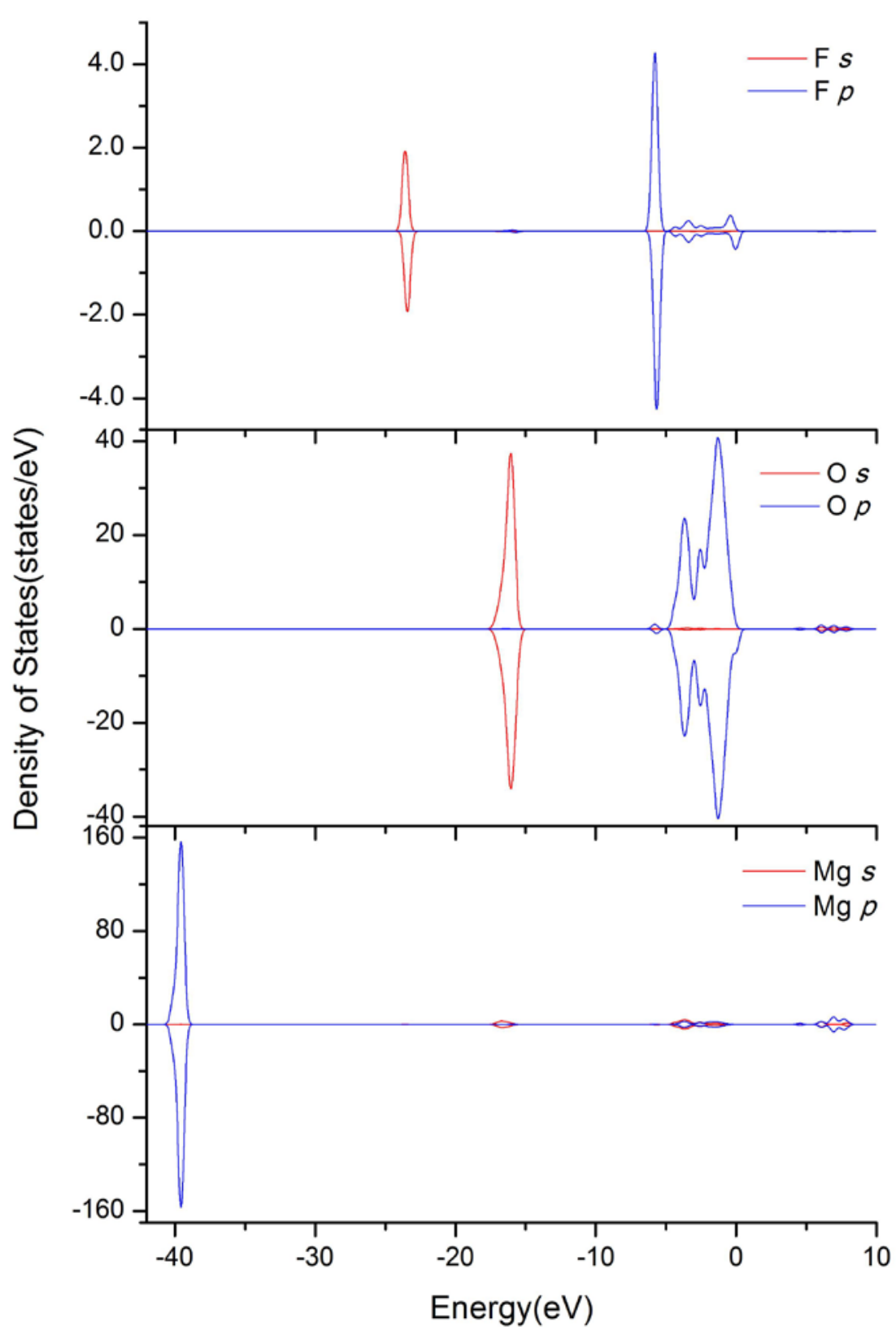

FIG. 1. Density of States of rocksalt MgO supercells of $2 \times 2 \times 2$ with fluorine interstitial. The interstitial positions are tetrahedral $(T)$ sites.

The spin-resolved band structures of the supercell are given in Fig. 2. It can be seen that the incorporation of $\mathrm{F}$ introduces an impurity gap state, with both the majority and minority spin components retaining a band gap. It shows a half-metallic behavior with the majority spins being semiconducting while the minority spins being metallic with sufficient unfilled states above the Fermi level. These unfilled states behave like free holes, although slightly localized. Based on the calculated band structures and charge population analysis, we propose hole mediation as the mechanism of ferromagnetic coupling in F-doped these oxides. The holes can be introduced by $\mathrm{F}$ interstitials $2 p$ states, then couple with the parent $\mathrm{O} 2 p$ states by a $p$ - $p$ interaction, similar to $p$ - $d$ hybridization in TM-doping. This $p$ - $p$ interaction leads to the appearance of additional localized mixed band levels derived from the valence band. The coupling pushes the minority $p-p$ mixed state upward and the opposite spin state downward, lowering the total energy of the system. Furthermore, it can be seen that there are three bands across the Fermi level, which imply the existence of $p$-like triplet state. Similarly for cation vacancy, the occurrence of a magnetic Jahn-Teller distortion stabilizes the spin singlet to the triplet state otherwise expected from the high symmetry crystal field. ${ }^{24}$ 

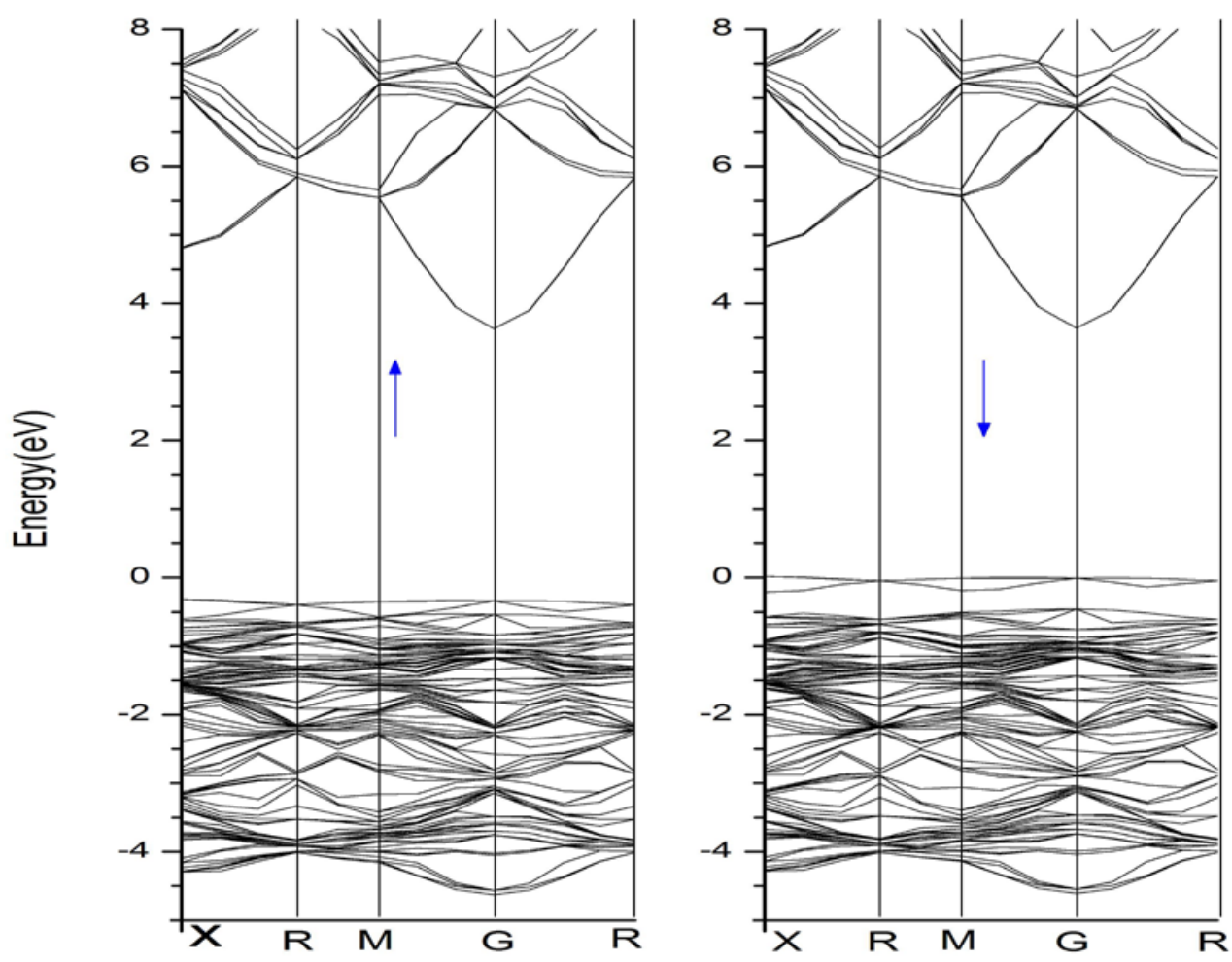

FIG. 2. Band Structures of rocksalt MgO supercells of $2 \times 2 \times 2$ with fluorine interstitial. The up-arrow denotes the majority-spin band, and the down-arrow the minority-spin band.

In conclusion, our calculations predicted ferromagnetism in fluorine-doped $\mathrm{MgO}$ and revealed that it results from fluorine interstitials. The intrinsic ferromagnetism originates from the F-O system in $\mathrm{MgO}$ environment. Our work demonstrates that fluorine is a novel dopant in the class of doped group-II oxides dilute magnetic materials.

\section{References}

[1]H. Ohno, Science 281, 951 (1998).

[2]P. Sharma, A. Gupta, K. V. Rao, F. J. Owens, R. Sharmaa, R. Ahuja, J. M. O. Guillen, B. Johansson, and G. A. Gehring, Nature Mater. 2, 673 (2003).

[3]J. M. D. Coey, A. P. Douvalis, C. B. Fitzgerald, and M. Venkatesan, Appl. Phys. Lett. 84, 1332 (2004).

[4]J. Philip, N. Theodoropoulou, G. Berera, and J. S. Moodera, Appl. Phys. Lett. 85, 777 (2004).

[5]A. Gupta, H. Cao, K. Parekh, K. V. Raoa, A. R. Raju, and U. V. Waghmare, J. Appl. Phys. 101, 09N513 (2007).

[6]J. W. Quilty, A. Shibata, J. Y. Son, K. Takubo, T. Mizokawa, H. Toyosaki, T. Fukumura, and M. Kawasaki, Phys. Rev. Lett. 96, 027202 (2006).

[7]H. Raebiger, S. Lany, and A. Zunger, Phys. Rev. B 79, 165202 (2009).

[8]X. Y. Cui, J. E. Medvedeva, B. Delley, A. J. Freeman, N. Newman, and C. Stampfl, Phys. Rev. Lett. 95, 256404 (2005).

[9]T. C. Kaspar, T. Droubay, S. M. Heald, M. H. Engelhard, P. Nachimuthu, and S. A. Chambers, Phys. Rev. B 77, 201303® (2008).

[10]S. Q. Zhou, K. Potzger, J. von Borany, R. GrÖtzschel, W. Skorupa, M. Helm, and J. Fassbender, Phys. Rev. B 77, 035209 (2008).

[11]M. Venkatesan, C. B. Fitzgerald, and J. M. D. Coey, Nature (London) 430, 630 (2004).

[12]M. W. Zhao, F. C. Pan, and L. M. Mei, Appl. Phys. Lett. 96, 012508 (2010). 
[13]R. Monnier and B. Delley, Phys. Rev. Lett. 87, 157204 (2001).

[14]I. S. Elfimov, S. Yunoki, and G. A. Sawatzky, Phys. Rev. Lett. 89, 216403 (2002).

[15]L. S. Dorneles, M. Venkatesan, M. Moliner, J. G. Lunney, and J. M. D. Coey, Appl. Phys. Lett. 85, 6377 (2004).

[16]W. W. Lei, D. Liu, P. W. Zhu, X. H. Chen, Q. Zhao, G. H. Wen, Q. L. Cui, and G. T. Zou, Appl. Phys. Lett. 95, 162502 (2009).

[17]R. Monnier and B. Delley, Phys. Rev. Lett. 87, 157204 (2001).

[18]M. D. Segall, P. J. D. Lindan, M. J. Probert, C. J. Pickard, P. J. Hasnip, S. J. Clark, and M. C. Payne, J. Phys.: Condens. Matter 14, 2717 (2002).

[19]J. P. Perdew, K. Burke, and M. Ernzerhof, Phys. Rev. Lett. 77, 3865 (1996).

[20]J. Monkhorst and J. Pack, Phys. Rev. B 13, 5188 (1976).

[21]P. Karen, A. Kjekshus, Q. Huang, and V. L. Karen, J. Alloys Compd. 282, 72 (1999).

[22]T. Brudevoll, E. A. Kotomin, and N. E. Christensen, Phys. Rev. B 53, 7731 (1996).

[23]C. A. Gilbert, S. D. Kenny, R. Smith, and E. Sanville, Phys. Rev. B 76, 184103 (2007).

[24]A. Zywietz, J. Furthmüller, and F. Bechstedt, Phys. Rev. B 62, 6854 (2000). ${ }^{80}$ A. Zywietz, J.

Furthmüller, and F. Bechstedt, Phys. Rev. B 62, 6854 (2000). 